\title{
RETURN TO WORK ITS IMPACT ON THE QUALITY OF LIFE OF BRAZILIAN BREAST CANCER PATIENTS
}

Isabela C. F. Colombino', Idam Oliveira-Junior ${ }^{1}$, Fabiola C. B. Silva', Jonathas J. Silva', Almir J. Sarri', Rene A. C. Vieira'

${ }^{1}$ Hospital de Câncer de Barretos, Fundação Pio XII - Barretos (SP), Brazil.

Objective: To evaluate the impact of return to work on the quality of life of breast cancer patients, and factors related to non- return to work. Method: A prospective, cross-sectional study approved by the Research Ethics Committee (1180/2016). The study was performed in patients who worked in the during 2012-2014; clinical stage 0 to III, age 25-60 years old. Based on a previous National study, a sample of 304 women was estimated. Standardized clinical records evaluated all patients, and the questionnaire EORTC QLQ-C30, EORTC BR-23, SPADI and HADS were also applied. Patients were randomized to a 1:1 to perform physical therapy examination, which was based on shoulder goniometry, hand dynamometry, and limb volume. Data were analyzed by the chi-square test and the Mann-Whitney test. Univariate and multivariate analysis were performed. Results: 304 were included, where 163 were submitted to physiotherapy evaluation. The mean age and schooling was 46.1 years and 11 years, respectively. $49.3 \%$ underwent conservative surgery; $51.5 \%$ axillary lymphadenectomy; $86.1 \%$ received chemotherapy, $84.2 \%$ had adjuvant hormone therapy, and $87.7 \%$ had radiotherapy, but $46.2 \%$ had radiotherapy on the supraclavicular fossa. At the moment of the diagnosis $50.7 \%$ exerted manual activities, $28.0 \%$ intellectual activities; and 21.4\% manual and intellectual activities. 94.7\% stopped working during treatment. 84.9\% received social security benefits. $54.0 \%$ returned to work after treatment. The women who returned to work presented lower age $(\mathrm{p}<0.001)$, higher schooling $(\mathrm{p}<0.0001)$, higher income $(\mathrm{p}<0.001)$, and smaller initial tumor size $(\mathrm{p}=0.008)$. These patients were generally submitted to sentinel lymph node $(\mathrm{p}=0.02)$, received adjuvant chemotherapy ( $\mathrm{p}=0.004)$, and had intellectual demand work $(p<0.001)$. There was an overall significant decrease in work capacity. Patients whose had loss of strength in the hand had a 2.2-fold risk of non-return to work $(\mathrm{p}=0.02)$. In the multivariate model to evaluate the not return to work, pre-treatment variables were age, schooling and clinical staging. Before treatment, lymphadenectomy increased 1.9 times the risk of not returning to work, and self-reported loss of strength in the hand 2.9 times. Physical demand work has raised the risk by 2.7 times. The women who returned to work presented a better quality of life through the EORTC QLQ C30 questionnaire; by BR23 presented higher scores related to body image and sexual function; by SPADI lower scores in relation to disability and pain; and by HADS lower scores related to anxiety and depression. Conclusion: $54 \%$ of patients did not return to work after breast cancer treatment. It was influenced by age, schooling, previous type of activity, axillary treatment, and physical sequel related to loss of strength on the hand. Return to work improved patients' quality of life. 